\title{
A synonymous codon change in the LMNA gene alters mRNA splicing and causes limb girdle muscular dystrophy type 1B
}

\author{
A Todorova, B Halliger-Keller, M C Walter, M-C Dabauvalle, H Lochmüller, C R Müller
}

J Med Genet 2003;40:e1 15 (http://www.jmedgenet.com/cgi/content/full/40/10/e115)

T he autosomal dominant form of Emery-Dreifuss muscular dystrophy (EDMD2; OMIM \# 181350) was the first disorder to be associated with mutations in the LMNA gene, encoding the nuclear envelope proteins lamin A and C. Following this, other mutations were reported in limb girdle muscular dystrophy with atrioventricular conduction defects (LGMDIB; OMIM \#159001) ${ }^{2}$ and a conduction system disease with dilated cardiomyopathy (CMDIA; OMIM \#115200). ${ }^{3}$ These disorders are reminiscent of EDMD2, with a predominance of skeletal and cardiac symptoms, respectively, but lacking the early contractures of large joints typical of EDMD. Four more entirely different phenotypes can also be caused by the LMNA gene: (a) partial lipodystrophy (FPLP; OMIM \#151660), ${ }^{4}$ (b) an autosomal recessive variant of Charcot-Marie-Tooth disorder type 2 (CMT2Bl; OMIM \#605588), ${ }^{5}$ (c) mandibuloacral dysplasia (MAD; OMIM \#248370 $)^{6}$ and (d) Hutchinson-Gilford progeria syndrome (HGPS; OMIM \# 176670). ${ }^{7}$ To date, more than 30 mutations have been reported to be associated with the muscular dystrophy phenotype. The majority of these mutations are missense substitutions, but a single case each of a nonsense substitution and a splice site mutation as well as a few small deletions are on record (Leiden Muscular Dystrophy pages, www.dmd.nl). Very recently, reports on mutations that activate cryptic splice sites have been found in the Hutchinson-Gilford progeria syndrome. ${ }^{78}$

Here, we report on a synonymous codon change in the LMNA gene, which leads to abnormal splicing and is likely to cause LGMDIB in a large German pedigree. Such "neutral" synonymous codon changes leading to splicing disorganisation have been described for the hexoseaminidase $\mathrm{A},{ }^{9}$ calpain $3,{ }^{10} F G F R 22^{11}$ and fibrillin-1 genes. ${ }^{12}$ Here, we present evidence that the replacement of the last nucleotide in exon 2 of the $L M N A$ gene $(\mathrm{c} .513 \mathrm{G} \rightarrow \mathrm{A})$ leads to partial skipping of the canonical $5^{\prime}$ splice site in intron 2 and the alternative use of a cryptic GT donor site within that intron. Our findings again stress the need to interpret such apparently "neutral" nucleotide changes with caution.

\section{MATERIALS AND METHODS}

The family described is of German descent, and there is no consanguinity. The father of the index patient was probably affected, but was not examined by us. He died at the age of 48 years from cardiac complications. Three sisters and the patient's son showed similar symptoms of mild muscle weakness, cardiac abnormalities, and hyperlordosis; one brother and the daughter of the index patient were not affected. The pedigree analysis is compatible with an autosomal dominant trait of inheritance. The index patient had normal developmental milestones, and did not complain of muscle weakness during childhood. Symptoms at onset, at the age of 14 years, were mild proximal weakness during longer walks. At first examination by us at at the age of 56

\section{Key points}

- We report on a synonymous codon change in the LMNA gene in a large German pedigree, which leads to abnormal splicing and is likely to cause limb girdle muscular dystrophy type 1B.

- The effect of the detected synonymous codon change was verified at the mRNA level by cloning and sequencing of the obtained transcription products. The replacement of the last nucleotide in exon 2 (c.513G $\rightarrow$ A) leads to partial skipping of the canonical $5^{\prime}$ splice site in intron 2 and the alternative use of a cryptic GT donor site within intron 2.

- It is clear that so-called "neutral" mutations in the human genome are not such rare events, and before considering them as neutral or non-pathogenic polymorphisms, their impact on mRNA processing should be assessed.

years, there was a moderate proximal weakness of $4 / 5$ on the MRC muscle strength grading scale in the biceps brachii and quadriceps muscles. The patient showed mild scapular winging and walked with hyperlordosis. No contractures of muscles and joints or severe muscle atrophy were detectable. Creatine kinase levels were normal on several occasions. The index patient (aged 56 years and three sisters (one aged 51 years, two aged 54 years) had cardiac pacemakers implanted because of conduction defects. Electromyographic findings of affected muscles were compatible with chronic myopathy. A diagnostic muscle biopsy was performed and showed a mild degenerative myopathy without inflammatory, specific structural or neurogenic changes on standard histological examinations.

The index patient approached us for genetic analysis. Additionally, four other members of the family (three affected and one non-affected) (fig 1) allowed testing for the synonymous codon substitution observed in the index patient.

\section{METHODS}

\section{Mutation screening}

Leukocyte DNA of the index patient was screened for mutations in the LMNA gene. All 12 exons of the gene, including the exon/intron splice sites, were amplified by PCR and directly sequenced by the CEQ2000 Dye Terminator Cycle

Abbreviations: $\mathrm{CV}$, consensus value 
Sequencing with Quick Start Kit (Beckman Coulter, Krefeld, Germany). The primers used for amplification and sequencing of exon 2 were: forward $5^{\prime}$-GACTCCTTCTCTTAAATCTA CTCTCC-3' and reverse 5'-GGGAGGGCCTAGGTAGAAGA-3'.

\section{Reverse transcriptase-polymerase chain reaction}

Total RNA was extracted from a fresh blood sample of the index patient to check the effect of the detected synonymous codon change at the mRNA level. Reverse transcription was performed with Superscript (Invitrogen, Karlsruhe, Germany) and a standard oligo-dT primer, using the manufacturer's protocol. cDNA was amplified with primers in exon 2 (2forward 5'-GGAGGGTGACCTGATAGCTG-3') and in exon 3 (3reverse $5^{\prime}$-GTCCAGTTCCTCCTTCATGG-3'). The obtained two amplification fragments (fig 2B) were cloned using the TOPO TA cloning kit (Invitrogen) and sequenced with the same primers as in the amplification reaction.

We checked 100 control chromosomes for the observed base substitution by single strand conformation analysis.

\section{RESULTS AND DISCUSSION}

All twelve exons and the flanking intronic sequences of the LMNA gene were screened for mutations in the index patient by direct sequencing. The only genomic sequence variation we detected (fig 2A) was an apparently silent third position codon change in exon 2: c.513G $\rightarrow$ A, Lys(AAG) 171 Lys(AAA). Four additional family members, three affected and one nonaffected, were available to study the segregation of this codon change in the family. All affected members were heterozygous for the substitution in exon 2 as was the index patient himself. The non-affected individual did not carry this nucleotide change (fig 1) nor was the sequence change found in DNA from 50 control individuals.

Because the mutation affected the last nucleotide of exon 2 , we speculated that it could possibly affect the splicing efficiency. Reverse transcription was performed on leukocyte mRNA from the index patient, and cDNA was amplified with primers in exon 2 and 3, in order to surround the region of suspected splice alterations. A larger than normal transcription product was observed at about $25 \%$ the intensity of the normal one (fig 2B). The corresponding PCR fragments were cloned and sequenced. The results obtained for the abnormally sized fragment showed that 45 nucleotides from intron 2 were included in the cDNA sequence between exon 2 and exon 3 (fig 2C). Thus, an additional 15 amino acids (VRPPCRAHPWPHLTH) are inserted into the protein. This is likely to change the protein conformation dramatically. In leukocytes, this substitution reduces the splicing efficiency of the intron 2 splice donor site and leads to partial use of a cryptic splice site 45 nucleotides downstream in intron 2 . From a comprehensive survey of primate genes, a splice site consensus sequence has been derived (AGgtaagt), and the relative impact of all nucleotide positions involved $\left(5^{\prime} \rightarrow-2\right.$, $\left.-1,+1,+2,+3,+4,+5,+6 \rightarrow 3^{\prime}\right)$ has been calculated to give a "consensus value" $(\mathrm{CV})^{13}{ }^{14}$, which allows for an estimate of the relative splicing efficiency for any deviation from the consensus sequence. In our case, the calculated CVs for the normal splice site $(\mathrm{CVN})$ and for the mutant splice site (CVM) were 0.921 and 0.797 respectively. The CV of the activated cryptic splice site (CVA) was 0.555 . As our mutation affects the -1 position of the consensus sequence, it is expected to have a dramatic effect on the splicing mechanism.

There are several pieces of evidence that support the notion that this mutation causes limb girdle muscular dystrophy in this family. First, it segregates with the disease in the available pedigree branch, which is compatible with autosomal dominant transmission. Secondly, cDNA from leukocytes showed two transcription products, one normal

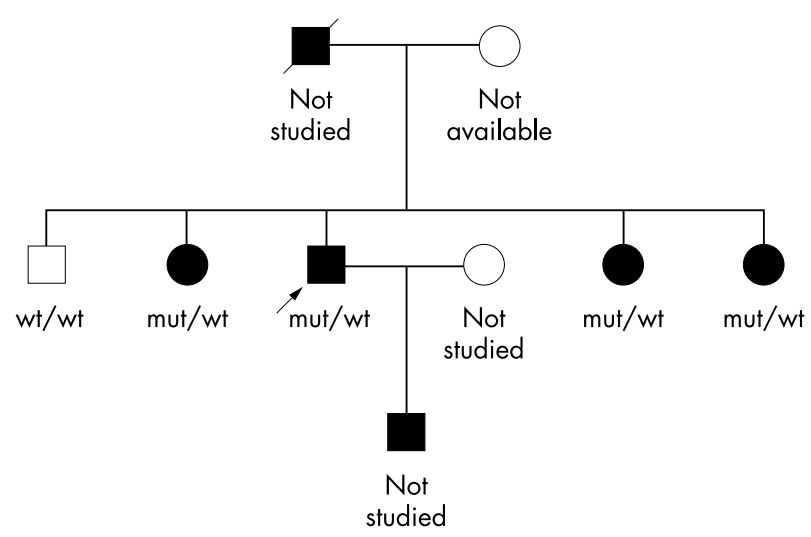

Figure 1 The pedigree of the studied family with limb girdle muscular dystrophy type 1B. The index patient is indicated by an arrow. The genotype of each proband is provided under the symbol: mut, mutant; wt, wild type.

and one larger, demonstrating that some abnormal splicing had occurred. The base substitution in the last nucleotide of exon 2 partially blocks the GT donor splice site of intron 2,

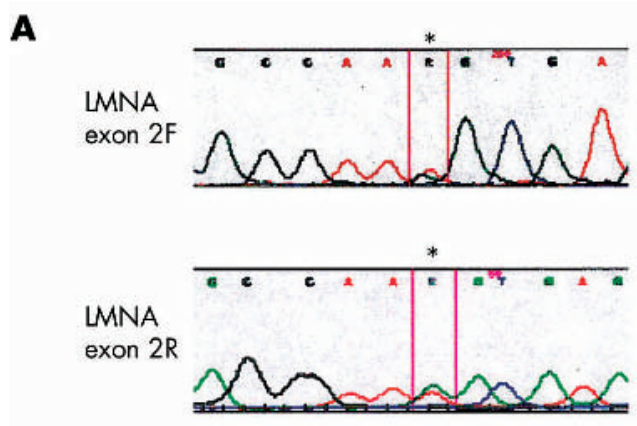

B

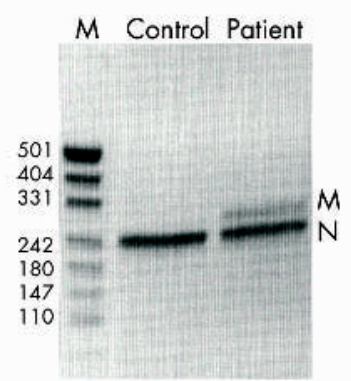

C

Exon 2

Exon 3

5'-GTGGCCAAAgtgaggecacectgeagggeceacecatggececacetaacacat CTTGAGGCA-3' $5 \cdot--V$
$513 G>A$

Figure 2 (A) Sequence of the LMNA exon 2 genomic DNA flanking the c. 513G $\rightarrow A$ transition ( $R$ asterisked) from the index patient. (B) Agarose electrophoresis of the RT-PCR products obtained from leukocyte mRNA of the index patient and a control sample. On right-hand side: $M$, mutant fragment; $N$, normal fragment. Lane $M$ is a marker: pUC19/Mspl (MBI Fermentas). (C) The sequence of the abnormally spliced product. Exonic parts are shown in capital letters, retained nucleotides of the intron are in small letters. The position of the c.513G $\rightarrow$ A substitution is indicated by an arrow and the mutant $A$ is given in bold. The newly inserted amino acids are provided under the corresponding codons. 
causing the first GT dinucleotide that follows along the intronic sequence to be recognised, and splicing is performed there. Given the relative abundance of this transcript it is possible that sufficient aberrant protein is produced to cause the disease. Thirdly, the sequence variant was not found in 100 control chromosomes and is, therefore, unlikely to be a polymorphism.

Lamin $\mathrm{A} / \mathrm{C}$ mutations cause a wide range of clinical phenotypes. ${ }^{15}$ While classical EDMD presents with early contractures, cardiomyopathy with conduction defects and muscle weakness, oligosymptomatic phenotypes have been described as "pure" cardiomyopathies (CMDl) or limb girdle muscular dystrophies with conduction disturbances (LGMDIB). Notably, contractures were absent in our patient and affected family members. Moreover, symptoms of muscular dystrophy started in early adulthood with mild limb girdle weakness, showed little progress, and did not lead to a loss of ambulation up to the seventh decade. The dominant and presenting symptom in all affected family members was cardiomyopathy with conduction defects, requiring pacemaker implantation in the fifth decade of life. LMNA mutations in pedigrees with a similar phenotype (LGMDIB) have been described previously. Interestingly, in one of three families, a splicing defect of lamin $\mathrm{A} / \mathrm{C}$ was detected, while the others showed missense mutations. ${ }^{2}$

Our results add more data regarding apparently "neutral" mutations in the human genome. Possibly, they are not such rare events as the few published reports suggest. ${ }^{-12}$ Before considering them as neutral or non-pathogenic polymorphisms, their impact on mRNA processing should be assessed.

\section{ACKNOWLEDGEMENTS}

A fellowship from the Alexander von Humboldt Foundation to A Todorova is gratefully acknowledged. This project is part of the German Muscular Dystrophy network (MD-NET, http:// www.netzwerk-muskeldystrophie.de).

\footnotetext{
Authors' affiliations

A Todorova, B Halliger-Keller, C R Müller, Department of Human Genetics, Biozentrum, University of Wuerzburg, Germany A Todorova, M-Ch Dabauvalle, Department of Cell and Developmental Biology, Biozentrum, University of Wuerzburg, Germany M C Walter, H Lochmüller, Friedrich-Baur-Institute, Dept. of Neurology and Gene Center Munich, University of Munich, Germany
}

Correspondence to: Professor Clemens R Mueller, Department of Human Genetics, Biozentrum, University of Wuerzburg, Am Hubland, Wuerzburg 97074, Germany; crm@biozentrum.uni-wuerzburg.de

\section{REFERENCES}

1 Bonne G, Di Barletta MR, Varnous S, Becane HM, Hammouda EH, Merlini L, Muntoni F, Greenberg CR, Gary F, Urtizberea JA, Duboc D, Fardeau M, Toniolo D, Schwartz K. Mutations in the gene encoding lamin A/C cause autosomal dominant Emery-Dreifuss muscular dystrophy. Nat Genet 1999:21:285-8.

2 Muchir A, Bonne G, van der Kooi AJ, van Meegen M, Baas F, Bolhuis PA, de Visser M, Schwartz K. Identification of mutations in the gene encoding lamins $\mathrm{A} / \mathrm{C}$ in autosomal dominant limb girdle muscular dystrophy with atrioventricular conduction disturbances (LGMDIB). Hum Mol Genet 2000:9:1453-9.

3 Fatkin D, MacRae C, Sasaki T, Wolff MR, Porcu M, Frenneaux M, Atherton J, Vidaillet HJ jR, Spudich S, De Girolami U, Seidman JG, Seidman C, Muntoni F, Muehle G, Johnson W, McDonough B. Missense mutations in the rod domain of the lamin $\mathrm{A} / \mathrm{C}$ gene as causes of dilated cardiomyopathy and conductionsystem disease. N Engl J Med 1999;341:1715-24.

4 Cao H, Hegele RA. Nuclear lamin A/C R482Q mutation in Canadian kindreds with Dunnigan-type familial partial lipodystrophy. Hum Mol Genet 2000;9:109-12.

5 De Sandre-Giovannoli A, Chaouch M, Kozlov S, Vallat JM, Tazir M, Kassouri N, Szepetowski P, Hammadouche T, Vandenberghe A, Stewart CL, Grid D, Levy N. Homozygous defects in LMNA, encoding lamin A/C nuclearenvelope proteins, cause autosomal recessive axonal neuropathy in human (Charcot-Marie-Tooth disorder type 2) and mouse. Am J Hum Genet 2002;70:726-36

6 Novelli G, Muchir A, Sangiuolo F, Helbling-Leclerc A, D'Apice MR, Massart C, Capon F, Sbraccia P, Federici M, Lauro R, Tudisco C, Pallotta R, Scarano G, Dallapiccola B, Merlini L, Bonne G. Mandibuloacral dysplasia is caused by a mutation in LMNA-encoding lamin A/C. Am J Hum Genet 2002;71:426-31.

7 Eriksson M, Brown WT, Gordon LB, Glynn MW, Singer J, Scott L, Erdos MR, Robbins CM, Moses TY, Berglund P, Dutra A, Pak E, Durkin S, Csoka AB, Boehnke M, Glover TW, Collins FS. Recurrent de novo point mutations in lamin A cause Hutchinson-Gilford progeria syndrome. Nature 2003;423:293-8.

8 De Sandre-Giovannoli A, Bernard R, Cau P, Navarro C, Amiel J, Boccaccio I, Lyonnet S, Stewart CL, Munnich A, Le Merrer M, Levy N. Lamin A truncation in Hutchinson-Gilford progeria. Science 2003;300:2055.

9 Akli S, Chelly J, Mezard C, Gandy S, Kahn A, Poenaru L. A "G" to "A" mutation at position -1 of a $5^{\prime}$ splice site in a late infantile form of Tay-Sachs disease. J Biol Chem 1990;265:7324-430.

10 Richard I, Beckmann JS. How neutral are synonymous codon mutations? Nat Genet 1995; 10:259.

11 Li X, Park WJ, Pyeritz RE, Jabs EW. Effect on splicing of a silent FGFR2 mutation in Crouzon syndrome. Nat Genet 1995;9:232-3.

12 Liu W, Qian Ch, Francke U. Silent mutation induces exon skipping of fibrillin-1 gene in Marfan syndrome. Nat Genet 1997;16:328-9.

13 Shapiro MB, Senapathy P. RNA splice junction of different classes of eukaryotes: sequence statistics and functional implications in gene expression. Nucleic Acids Res 1987;15:7155-74.

14 Krawczak M, Reiss J, Cooper DN. The mutational spectrum of single basepair substitutions in mRNA splice junctions of human genes: causes and consequences. Hum Genet 1992;90:41-54

15 Burke B, Stewart CL. Life at the edge: the nuclear envelope and human disease. Nat Rev Mol Cell Biol 2002;3:575-85. 